\title{
Nonlinear optical processes in optically trapped InP nanowires.
}

\begin{abstract}
We report on the observation of nonlinear optical excitation and related photoluminescence from single InP semiconductor nanowires held in suspension using a gradient force optical tweezers. Photoexcitation of free carriers is achieved through absorption of infrared $(1.17 \mathrm{eV})$ photons from the trapping source via a combination of two- and three-photon processes. This was confirmed by power-dependent photoluminescence measurements. Marked differences in spectral features are noted between nonlinear optical excitation and direct excitation and are related to band-filling effects. Direct observation of second harmonic generation in trapped InP nanowires confirms the presence of nonlinear optical processes.
\end{abstract}

Keyword: Semiconductor nanowires; Photoluminescence; Optical tweezers; Multi-photon absorption. 